\title{
Cumulants of Jack symmetric functions and $b$-conjecture (extended abstract)
}

\author{
Maciej Dołęga ${ }^{1,2} \mid \|^{\dagger}$ and Valentin Féray \\ ${ }^{1}$ Wydziat Matematyki i Informatyki, Uniwersytet im. Adama Mickiewicza, Umultowska 87, 61-614 Poznań, Poland, \\ ${ }^{2}$ Instytut Matematyczny, Uniwersytet Wrocławski, pl. Grunwaldzki 2/4, 50-384 Wrocław, Poland, \\ ${ }^{3}$ Institut für Mathematik, Universität Zürich, Winterthurerstrasse 190, 8057 Zürich, Switzerland
}

\begin{abstract}
Goulden and Jackson (1996) introduced, using Jack symmetric functions, some multivariate generating series $\psi(\boldsymbol{x}, \boldsymbol{y}, \boldsymbol{z} ; t, 1+\beta)$ that might be interpreted as a continuous deformation of the rooted hypermap generating series. They made the following conjecture: coefficients of $\psi(\boldsymbol{x}, \boldsymbol{y}, \boldsymbol{z} ; t, 1+\beta)$ are polynomials in $\beta$ with nonnegative integer coefficients. We prove partially this conjecture, nowadays called $b$-conjecture, by showing that coefficients of $\psi(\boldsymbol{x}, \boldsymbol{y}, \boldsymbol{z} ; t, 1+\beta)$ are polynomials in $\beta$ with rational coefficients. Until now, it was only known that they are rational functions of $\beta$. A key step of the proof is a strong factorization property of Jack polynomials when $\alpha \rightarrow 0$ that may be of independent interest.

Résumé. Goulden et Jackson (1996) ont introduit, en utilisant les fonctions symétriques de Jack, une série génératrice multivariée $\psi(\boldsymbol{x}, \boldsymbol{y}, \boldsymbol{z} ; t, 1+\beta)$ qui est une déformation continue de séries génératrices de cartes enracinées. Ils ont fait la conjecture suivante : les coefficients de $\psi(\boldsymbol{x}, \boldsymbol{y}, \boldsymbol{z} ; t, 1+\beta)$ sont des polynômes en $\beta$ avec des coefficients entiers positifs. Nous prouvons partiellemnt cette conjecture, en établissant la polynomialité en $\beta$ des coefficients de $\psi(\boldsymbol{x}, \boldsymbol{y}, \boldsymbol{z} ; t, 1+\beta)$ (ces coefficients sont a priori des fonctions rationnelles en $\beta$ ). Un ingrédient clé de notre preuve, potentiellement intéressant par ailleurs, est une propriété de factorisation forte des polynômes de Jack quand $\alpha$ tend vers 0 .
\end{abstract}

Keywords. Jack symmetric functions, Cumulants, Map enumeration, Laplace-Beltrami operator

This paper is an extended abstract of [2], which will be submitted elsewhere.

\section{Introduction}

\section{$1.1 \quad b$-conjecture and our main result}

Let $J_{\lambda}^{(\alpha)}(\boldsymbol{x})$ be the Jack symmetric function indexed by a partition $\lambda$ in the infinite alphabet $\boldsymbol{x}$. Let us denote by $h_{\alpha}(\lambda)$ and $h_{\alpha}^{\prime}(\lambda)$ the $\alpha$ hook-polynomials (these are combinatorial factors that appears often in Jack polynomial theory, and that are defined in Section 2.1. We also use $\mathcal{P}$ for the set of all integer

\footnotetext{
${ }^{\dagger}$ Acknowledges support from Agence Nationale de la Recherche, grant ANR 12-JS02-001-01 "Cartaplus", and from NCN, grant UMO-2015/16/S/ST1/00420.

‡Acknowledges support from SNF, grant "Dual combinatorics of Jack polynomials" SNF-149461.

1365-8050 @ 2016 Discrete Mathematics and Theoretical Computer Science (DMTCS), Nancy, France
} 
partitions and $|\lambda|$ for the size of a partition $\lambda$. In their article [6], Goulden and Jackson defined coefficients $h_{\mu, \nu}^{\tau}(\alpha-1)$ by the following formal series identity:

$$
\log \left(\sum_{\lambda \in \mathcal{P}} \frac{J_{\lambda}^{(\alpha)}(\boldsymbol{x}) J_{\lambda}^{(\alpha)}(\boldsymbol{y}) J_{\lambda}^{(\alpha)}(\boldsymbol{z}) t^{|\lambda|}}{h_{\alpha}(\tau) h_{\alpha}^{\prime}(\tau)}\right)=\sum_{n \geq 1} \frac{t^{n}}{\alpha n}\left(\sum_{\mu, \nu, \tau \vdash n} h_{\mu, \nu}^{\tau}(\alpha-1) p_{\mu}(\boldsymbol{x}) p_{\nu}(\boldsymbol{y}) p_{\tau}(\boldsymbol{z})\right),
$$

where $\mu, \nu, \tau \vdash n$ means that $\mu, \nu$ and $\tau$ are three partitions of $n$ and $p_{\mu}$ is the power-sum symmetric function associated with $\mu$.

This rather involved definition is motivated by the following combinatorial interpretations for particular values of $\alpha$; see [6, Section 1.1] and references therein.

- In the case $\alpha=1$, the quantity $h_{\mu, \nu}^{\tau}(0)$ enumerates connected hypergraphs embedded into oriented surfaces with vertex-, edge- and face-degree distributions given by $\mu, \nu$ and $\tau$.

- In the case $\alpha=2$, the quantity $h_{\mu, \nu}^{\tau}(1)$ enumerates connected hypergraphs embedded into non-oriented surfaces with the same degree conditions.

Connected hypergraphs embedded into surfaces are usually called maps and are a classical topic in enumerative combinatorics related to the computation of matrix integrals or the study of moduli spaces of curves, as explained in detail in the book [10]. The logarithm in Eq. (11) is present because we only want to count connected objects.

Note that $h_{\mu, \nu}^{\tau}(\alpha-1)$ is a priori a quantity depending on parameter $\alpha$, and describing it as a quantity depending on a different parameter $\beta:=\alpha-1$ might seem be artificial. However, it turned out that this shift seems to be a right one for finding a combinatorial interpretation of $h_{\mu, \nu}^{\tau}(\beta)$, as suggested by Goulden and Jackson [6] in the following conjecture.

Conjecture 1.1 (b-conjecture) For all partitions $\tau, \mu, \nu \vdash n \geq 1$, the quantity $h_{\mu, \nu}^{\tau}(\beta)$ is a polynomial in $\beta$ with nonnegative, integer coefficients. Moreover, there exists a statistics $\eta$ on maps such that

$$
h_{\mu, \nu}^{\tau}(\beta)=\sum_{\mathcal{M}} \beta^{\eta(\mathcal{M})},
$$

where the summation index runs over all rooted hypermaps $\mathcal{M}$ vertex-, edge-and face-degree distributions given by $\mu, \nu$ and $\tau$, and $\eta(\mathcal{M})$ is a nonnegative integer equals to 0 if and only if $\mathcal{M}$ is orientable.

This conjecture is still open. Some constructions for a candidate statistics $\eta$ have been given, establishing particular cases of the conjecture [1, 9, 7]. However, there is not much known about the structure of $h_{\mu, \nu}^{\tau}(\beta)$ for arbitrary partitions $\tau, \mu, \nu \vdash n$. Strictly from the construction it is only known to be rational function in $\beta$ with rational coefficients. It follows from recent results of us [3, Appendix B] that the only possible pole of this rational function is at $\beta=-1$ (i.e. $\alpha=0$ ); see the long version [2] for details. Our main result in this paper is that there is no pole at $\beta=-1$ either, completing the proof of the polynomiality of $h_{\mu, \nu}^{\tau}(\beta)$ for all partitions $\tau, \mu, \nu \vdash n \geq 1$. This gives more evidence towards Conjecture 1.1. and we believe that it opens some new possible ways to prove it in full generality (such as polynomial interpolation, for instance).

Theorem 1.2 For all partitions $\tau, \mu, \nu \vdash n \geq 1$, the quantity $h_{\mu, \nu}^{\tau}(\beta)$ has no pole in $\beta=-1$ and hence is a polynomial in $\beta$ (with rational coefficients).

Unfortunately, the nonnegativity and the integrality of the coefficients seem out of reach with our approach. 


\subsection{Strong factorization of Jack polynomials}

A key step in our proof of Theorem 1.2 is a strong factorization of Jack polynomials when $\alpha$ tends to zero. Unfortunately it is hard to explain at this stage how this result enters the proof of our main result. Nevertheless, since we think that it could also be interesting in itself, let us present this intermediate result in the introduction.

To state it let us introduce a few notations. If $\lambda$ and $\mu$ are partitions, we define $\lambda \oplus \mu:=\left(\lambda_{1}+\mu_{1}, \lambda_{2}+\right.$ $\left.\mu_{2}, \ldots\right)$ their entry-wise sum. If $\lambda^{1}, \cdots, \lambda^{r}$ are partitions and $I$ a subset of $[r]:=\{1, \cdots, r\}$, then we denote $\lambda^{I}:=\bigoplus_{i \in I} \lambda^{i}$.

Theorem 1.3 Let $r \geq 2$ be an integer and $\lambda^{1}, \cdots, \lambda^{r}$ be partitions. Then

$$
\prod_{I \subset[r]}\left(J_{\lambda^{I}}^{(\alpha)}\right)^{(-1)^{|I|}}=1+O\left(\alpha^{r-1}\right) .
$$

For a formal definition of $O\left(\alpha^{r}\right)$ in this context, we refer the reader to Definition 3.1.

For $r=2$, the theorem asserts that $J_{\lambda^{1} \oplus \lambda^{2}}^{(\alpha)}=J_{\lambda^{1}}^{(\alpha)} J_{\lambda^{2}}^{(\alpha)}(1+O(\alpha))$, i.e. that $J_{\lambda^{1} \oplus \lambda^{2}}^{(\alpha)}$ factorizes when $\alpha$ tends to 0 . This follows from the explicit expression of Jack polynomials for $\alpha=0$ [15, Proposition 7.6]. For bigger values of $r$, the theorem is much more involved and can be interpreted as a strong factorization property for Jack polynomials.

The theorem has an equivalent form that uses the notion of cumulants of Jack polynomials - see Section 3 for comments on the terminology. For partitions $\lambda^{1}, \cdots, \lambda^{r}$, we denote

$$
\kappa^{J}\left(\lambda^{1}, \cdots, \lambda^{r}\right)=\sum_{\pi \in \mathcal{P}([r])}(-1)^{\#(\pi)-1}(\#(\pi)-1) ! \prod_{B \in \pi} J_{\lambda^{B}}^{(\alpha)} .
$$

Here, the sum is taken over set-partitions $\pi$ of $[r]$ and $\#(\pi)$ is the number of blocks of a set-partition $\pi$. For example

$$
\begin{aligned}
\kappa^{J}\left(\lambda^{1}, \lambda^{2}\right) & =J_{\lambda^{1} \oplus \lambda^{2}}^{(\alpha)}-J_{\lambda^{1}}^{(\alpha)} J_{\lambda^{2}}^{(\alpha)}, \\
\kappa^{J}\left(\lambda^{1}, \lambda^{2}, \lambda^{3}\right) & =J_{\lambda^{1} \oplus \lambda^{2} \oplus \lambda^{3}}^{(\alpha)}-J_{\lambda^{1}}^{(\alpha)} J_{\lambda^{2} \oplus \lambda^{3}}^{(\alpha)}-J_{\lambda^{2}}^{(\alpha)} J_{\lambda^{1} \oplus \lambda^{3}}^{(\alpha)}-J_{\lambda^{3}}^{(\alpha)} J_{\lambda^{1} \oplus \lambda^{2}}^{(\alpha)}+2 J_{\lambda^{1}}^{(\alpha)} J_{\lambda^{2}}^{(\alpha)} J_{\lambda^{3}}^{(\alpha)} .
\end{aligned}
$$

We then have the following estimate for cumulants of Jack polynomials

Theorem 1.4 Let $r \geq 2$ be an integer and $\lambda^{1}, \cdots, \lambda^{r}$ be partitions. Then

$$
\kappa^{J}\left(\lambda^{1}, \cdots, \lambda^{r}\right)=O\left(\alpha^{r-1}\right) .
$$

Theorem 1.4 is in fact equivalent to Theorem 1.3, as shown (in a more general setting) by Proposition 3.3 (we need here the fact that $J_{\lambda}^{(\alpha)}$ has a non-zero limit when $\alpha$ tends to 0 [15, Proposition 7.6]; this ensures that $J_{\lambda}^{(\alpha)}=O(1)$ and $\left(J_{\lambda}^{(\alpha)}\right)^{-1}=O(1)$ ). We prove Theorem 1.4 in Section 4

A similar strong factorization property conjecturally holds for Macdonald polynomials, see [2].

\subsection{Related problems}

We finish this section by mentioning two similar problems. First, a very similar conjecture to Conjecture 1.1 without taking a logarithm in Eq. (1) was also stated by Goulden and Jackson [6]. The latter is conjecturally a multivariate generating series of matchings, where the exponent of $\beta$ is some combinatorial 
integer-valued statistics. The conjecture is still open, while some special cases have been solved by Goulden and Jackson in their original article [6] and recently by Kanunnikov and Vassilieva [7]. The polynomiality was proven by the authors of this paper in [3] and is used to reduce the proof of Theorem 1.2 to checking that there is no singularity in $\alpha=0$.

A second related problem is the investigation of Jack characters, that is suitably normalized coefficients of power-sum symmetric function expansion of Jack polynomials. In a series of paper [11, 12], Lassalle made some polynomiality and positivity conjectures suggesting that a combinatorial description of these objects might exist. Although these conjectures are not fully resolved, it was proven by us together with Śniady [4] that in some special cases indeed, such combinatorial setup exists. Moreover, as in Conjecture 1.1 , these special cases involve hypermaps and some statistics that "measures their non-orientability".

We cannot resist to state that there must be a deep connection between all these problems, and understanding it would be of great interest.

\section{Preliminaries}

\subsection{Partitions}

We call $\lambda:=\left(\lambda_{1}, \lambda_{2}, \ldots, \lambda_{l}\right)$ a partition of $n$ if it is a weakly decreasing sequence of positive integers such that $\lambda_{1}+\lambda_{2}+\cdots+\lambda_{l}=n$. Then $n$ is called the size of $\lambda$ while $l$ is its length. As usual, we use the notation $\lambda \vdash n$, or $|\lambda|=n$, and $\ell(\lambda)=l$. We denote the set of partitions of $n$ by $\mathcal{Y}_{n}$, which we endow with the dominance order defined in the following way: $\lambda \leq \mu \Longleftrightarrow \sum_{i \leq j} \lambda_{i} \leq \sum_{i \leq j} \mu_{i}$ for any positive integer $j$. We also denote $\lambda^{t}$ the conjugate partition of $\lambda$. As usual, we identify partition with their Young diagrams (using French convention). For any box $\square:=(i, j) \in \lambda$ from Young diagram we define its arm-length by $a(\square):=\lambda_{j}-i$ and its leg-length by $\ell(\square):=\lambda_{i}^{t}-j$ (the same definitions as in [14, Chapter I]), see Figure 1 .

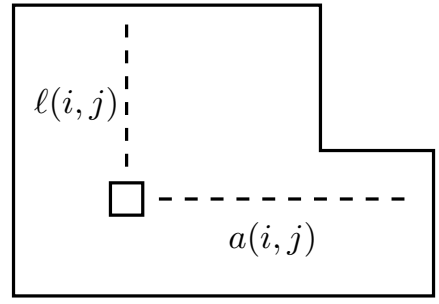

Fig. 1: Arm and leg length of boxes in Young diagrams.
There are many combinatorial quantities associated with partitions that we will use extensively through this paper. First, set

$$
z_{\lambda}:=\prod_{i \geq 1} i^{m_{i}(\lambda)} m_{i}(\lambda) !
$$

where $m_{i}(\lambda)$ denotes the number of parts of $\lambda$ equal to $i$. We also define $\alpha$-hook polynomials $h_{\alpha}(\lambda)$ and $h_{\alpha}^{\prime}(\lambda)$ by the following equations:

$$
\begin{aligned}
h_{\alpha}(\lambda) & :=\prod_{\square \in \lambda}(\alpha a(\square)+\ell(\square)+1), \\
h_{\alpha}^{\prime}(\lambda) & :=\prod_{\square \in \lambda}(\alpha a(\square)+\ell(\square)+\alpha) .
\end{aligned}
$$

Finally, we consider a partition binomial given by $b(\lambda):=\sum_{i}\left(\begin{array}{c}\lambda_{i} \\ 2\end{array}\right)$.

\subsection{Jack polynomials and Laplace-Beltrami operator}

Jack polynomials are a classical one-parameter deformation of Schur symmetric functions that can be defined in several different ways. To our purpose, we will use a characterization via Laplace-Beltrami operators, suggested by Stanley in his seminal paper [15] (note p. 85). Since this is now a well-established 
theory, results of this section are given without proofs but with explicit references to the literature (mostly to Stanley's paper [15]).

First, consider the vector space $\operatorname{Sym}^{N}$ of symmetric polynomials in $N$ variables over $\mathbb{Q}(\alpha)$. The following differential operators act on this space:

$$
D_{1}=\sum_{i \leq N} \sum_{i \neq j} \frac{x_{i}^{2}}{x_{i}-x_{j}} \frac{\partial}{\partial x_{i}}, \quad D_{2}=\frac{1}{2} \sum_{i \leq N} x_{i}^{2} \frac{\partial^{2}}{\partial x_{i}^{2}} .
$$

We define Laplace-Beltrami operator $D_{\alpha}$ as $D_{\alpha}=D_{1}+\alpha D_{2}$.

Proposition 2.1 There exists a unique family $J_{\lambda}^{(\alpha)}$ (indexed by partitions $\lambda$ of length at most $N$ ) in $\mathrm{Sym}^{N}$ that satisfy:

(C1) $J_{\lambda}^{(\alpha)}\left(x_{1}, \ldots, x_{N}\right)$ is an eigenvector of $D_{\alpha}$ with eigenvalue ev $(\lambda)=\left(\alpha b(\lambda)-b\left(\lambda^{t}\right)+(N-1)|\lambda|\right)$;

(C2) the monomial expansion of $J_{\lambda}^{(\alpha)}$ is given by

$$
J_{\lambda}^{(\alpha)}=h_{\alpha}(\lambda) m_{\lambda}+\sum_{\nu<\lambda} a_{\nu}^{\lambda} m_{\nu}, \text { where } a_{\nu}^{\lambda} \in \mathbb{Q}(\alpha) .
$$

(Recall that we use the dominance order on partitions.)

These polynomials are called Jack polynomials.

This is not the definition of Jack polynomials used by Stanley, but the fact that Jack polynomials indeed satisfy these properties can be found in [15]; see Theorem 3.1 and Theorem 5.6. The uniqueness is an easy linear algebra exercise when one has observed that $\operatorname{ev}(\lambda)=\operatorname{ev}(\mu)$ and $|\lambda|=|\mu| \operatorname{imply}$ that $\lambda$ and $\mu$ are either equal or incomparable for the dominance order [15, Lemma 3.2]. A deep result of Knop and Sahi [8] asserts that $a_{\nu}^{\lambda}$ lies in fact in $\mathbb{N}[\alpha]$. In particular, Jack polynomials depend polynomially on $\alpha$.

With the definition above, the Jack polynomial $J_{\lambda}^{(\alpha)}$ depends on the number $N$ of variables. However, it is easy to see that it satisfies the compatibility relation $J_{\lambda}^{(\alpha)}\left(x_{1}, \ldots, x_{N}, 0\right)=J_{\lambda}^{(\alpha)}\left(x_{1}, \ldots, x_{N}\right)$ and thus $J_{\lambda}^{(\alpha)}$ can be seen as a symmetric function. In the sequel, when working with differential operators, we sometimes confuse a symmetric function $f$ with its restriction $f\left(x_{1}, \ldots, x_{N}, 0,0, \ldots\right)$ to $N$ variables.

Stanley also established the following specialization formula at $\alpha=0: J_{\lambda}^{(0)}=\left(\prod_{i} \lambda_{i}^{t} !\right) e_{\lambda^{t}}$, where $e_{\lambda}$ is the elementary symmetric function associated with $\lambda$ [15, Proposition 7.6]. A key point in his proof, that will be also important in the present paper, is the following proposition.

Proposition 2.2 For any partition $\lambda \vdash n$,

1. the elementary symmetric function $e_{\lambda}$ is an eigenvector of the operator $D_{1}$ :

$$
D_{1} e_{\lambda}=((N-1)|\lambda|-b(\lambda)) e_{\lambda}
$$

2. for any partition $\mu \vdash n$ such that $b(\lambda)=b(\mu)$ either $\lambda=\mu$ or $\lambda \not \leq \mu$ in the dominance order.

Here is an easy corollary, whose proof is left as an exercise to the reader.

Corollary 2.3 Let $f \in$ Sym be a homogeneous symmetric function with an expansion in the monomial basis of the following form:

$$
f=\sum_{\boldsymbol{\mu}<\boldsymbol{\lambda}} d_{\mu} m_{\mu}
$$

for some partition $\lambda$. If, for any number $N$ of variables, $D_{1} f=\left((N-1)|\lambda|-b\left(\lambda^{t}\right)\right) f$ then $f=0$. 


\section{Strong factorization of hook products}

Let $R$ be a ring and $\alpha$ a formal parameter. Denote $R(\alpha)$ the field of rational function in $\alpha$ with coefficients in $R$. Throughout this paper, $\alpha$ is the Jack parameter.

Definition 3.1 We use the following notation: for $r \in R(\alpha)$ and an integer $k$, we write $r=O\left(\alpha^{k}\right)$ if the rational function $r \cdot \alpha^{-k}$ has no pole in 0 .

\subsection{Small cumulant and strong factorization property}

Recall that a set partition of a set $S$ is a (non-ordered) family of non-empty disjoint subsets of $S$ (called blocks of the partition), whose union is $S$. Denote $\mathcal{P}(S)$ the set of set partitions of a given set $S$.

Definition 3.2 Let $\left(u_{I}\right)_{I \subseteq J}$ be a family of elements in $R(\alpha)$, indexed by subsets of a finite set $J$. Then its partial cumulant is defined as follows. For any non-empty subset $H$ of $J$, set

$$
\kappa_{H}(\boldsymbol{u})=\sum_{\pi \in \mathcal{P}(H)}(-1)^{\#(\pi)-1}(\#(\pi)-1) ! \prod_{B \in \pi} u_{B} .
$$

The terminology comes from probability theory. Let $J=[r]$, and let $X_{1}, \cdots, X_{r}$ be random variables with finite moments defined on the same probability space. If $u_{I}=\mathbb{E}\left(\prod_{i \in I} X_{i}\right)$, where $\mathbb{E}$ denotes the expectation of this probability space, then the quantity $\kappa_{[r]}(\boldsymbol{u})$ is known as the joint (or mixed) cumulant of the random variables $X_{1}, \cdots, X_{r}$. Even if this probabilistic interpretation of cumulants is not relevant here, we will use several lemmas that have been discovered in a probabilistic context [5].

As above, we consider a family $\boldsymbol{u}=\left(u_{I}\right)_{I \subseteq[r]}$ of elements of $R(\alpha)$ indexed by subsets of $[r]$. We now assume that these elements are non-zero and $u_{\emptyset}=1$. We then define the cumulative factorization error terms $T_{H}(\boldsymbol{u})$ of the family $\boldsymbol{u}$. For any subset $H$ of $[r]$ of size at least 2 , set

$$
T_{H}(\boldsymbol{u})=\prod_{G \subseteq H} u_{G}^{(-1)^{|H|-|G|}}-1 .
$$

Proposition 3.3 Using the notation above, the following statements are equivalent:

I. Strong factorization property: for any subset $H \subseteq[r]$ of size at least 2 , one has

$$
T_{H}(\boldsymbol{u})=O\left(\alpha^{|H|-1}\right) .
$$

II. Small cumulant property: for any subset $H \subseteq[r]$ of size at least 2 , one has

$$
\kappa_{H}(\boldsymbol{u})=\left(\prod_{h \in H} u_{h}\right) O\left(\alpha^{|H|-1}\right) .
$$

This proposition is a reformulation of [5, Lemma 2.2]. For a proof in our context, which is an adaptation of the proof of [5, Lemma 2.2], we refer to the full version of this article [2, Proposition 3.3].

A first consequence of this multiplicative criterion for small cumulants is the following stability result.

Corollary 3.4 Consider two families $\left(u_{I}\right)_{I \subseteq[r]}$ and $\left(v_{I}\right)_{I \subseteq[r]}$ with the small cumulant property. Then their entry-wise product $\left(u_{I} v_{I}\right)_{I \subseteq[r]}$ and quotient $\left(u_{I} / v_{I}\right)_{I \subseteq[r]}$ also have the small cumulant property.

Proof: This is trivial for the strong factorization property and the small cumulant property is equivalent to it. 


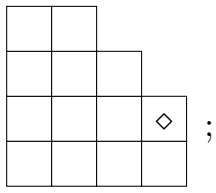

$\lambda^{1}$

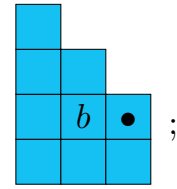

$\lambda^{2}$

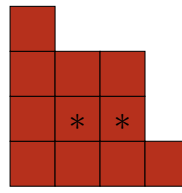

$\lambda^{3}$

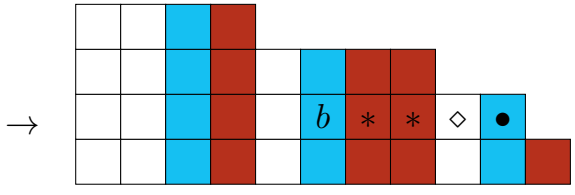

$\lambda^{1}+\lambda^{2}+\lambda^{3}$

Fig. 2: The diagram of an entry-wise sum of partitions.

\subsection{Hook cumulants}

To illustrate the propositions above and as a preparation for our next results, we show in this section that families constructed from the hook polynomials (6) and (7) have the small cumulant properties.

Lemma 3.5 Fix a positive integer $r$ and a subset $K$ of $[r]$. Let $C$ and $\left(c_{i}\right)_{i \in K}$ be some elements of $R(\alpha)$. Assume that $C, C^{-1}$ and the $c_{i}$ are $O(1)$. For a subset $I$ of $K$, we define $v_{I}=C+\alpha \cdot \sum_{i \in I} c_{i}$. Then, for any subset $H$ of $K$, we have $T_{H}(\boldsymbol{v})=O\left(\alpha^{|H|}\right)$.

This technical lemma is a reformulation of [5, Lemma 2.4] and, again, for a proof in our context, we refer to [2, Lemma 3.5]. We recall that for any fixed sequence of partitions $\lambda^{1}, \ldots, \lambda^{r}$ and for a subset $I \subset[r]$ we set $\lambda^{I}:=\bigoplus_{i \in I} \lambda^{i}$.

Proposition 3.6 Fix some partitions $\lambda^{1}, \ldots, \lambda^{r}$ and, for any subset I of $[r]$, set $u_{I}:=h_{\alpha}\left(\lambda^{I}\right)$. The family $\left(u_{I}\right)$ has the strong factorization, and hence, the small cumulant properties.

Proof: Clearly, it is enough to prove that $T_{[r]}(\boldsymbol{u})=O\left(\alpha^{r-1}\right)$.

Fix some subset $I=\left\{i_{1}, \cdots, i_{t}\right\}$ of $[r]$ with $i_{1}<\cdots<i_{t}$ and observe that the Young diagram $\lambda^{I}$ can be constructed by sorting the columns of the diagrams $\lambda^{i_{1}}, \ldots, \lambda^{i_{t}}$ in decreasing order of their length. When several columns have the same lengths, we put first the columns of $\lambda^{i_{1}}$, then those of $\lambda^{i_{2}}$ and so on; see Figure 2 (at the moment, please disregard symbols in boxes). This gives a way to identify boxes of $\lambda^{I}$ with boxes of the diagrams $\lambda^{i_{s}}(1 \leq s \leq t)$. With this identification, if $b=(r, c)$ is a box in $\lambda^{g}$ for some $g \in I$, its leg-length $\ell(b)$ in $\lambda^{I}$ is the same as in $\lambda^{g}$. At the opposite, the arm length of $b$ in $\lambda^{I}$ may be bigger than the one in $\lambda^{g}$. We denote these two quantities by $a_{I}(b)$ and $a_{g}(b)$, respectively. Let us also define, $a_{i}(b)$ for $i \neq g$ in $I$, as

- for $i<g, a_{i}(b)$ is the number of boxes $b^{\prime}$ in the $r$-th row of $\lambda^{i}$ such that the size of the column of $b^{\prime}$ is smaller than the size of the column of $b$ (e.g., on Figure 2 for $i=1$, these are boxes with a diamond);

- for $i>g, a_{i}(b)$ is the number of boxes $b^{\prime}$ in the $r$-th row of $\lambda^{i}$ such that the size of the column of $b^{\prime}$ is at most the size of the column of $b$ (e.g., on Figure 2 for $i=3$, these are boxes with an asterisk).

Looking at Figure 2 it is easy to see that $a_{I}(b)=\sum_{i \in I} a_{i}(b)$. Therefore, for $G \subseteq[r]$, one has:

$$
u_{G}=h_{\alpha}\left(\bigoplus_{g \in G} \lambda^{g}\right)=\prod_{g \in G}\left[\prod_{b \in \lambda^{g}} \ell(b)+1+\alpha \cdot a_{G}(b)\right] \text {. }
$$

Plugging it into Eq. (9) (which is the definition of $T_{[r]}(\boldsymbol{u})$ ) and changing the order of the products, we get:

$$
1+T_{[r]}(\boldsymbol{u})=\prod_{g \in[r]} \prod_{b \in \lambda^{g}}\left[\prod_{\substack{G \subseteq[r] \\ G \ni g}}\left(\ell(b)+1+\alpha \cdot a_{G}(b)\right)^{(-1)^{r-|G|}}\right] .
$$


The expression inside the bracket corresponds to $1+T_{[r] \backslash\{g\}}\left(\boldsymbol{v}^{b}\right)$, where $\boldsymbol{v}^{b}$ is defined as follows: if $I$ is a subset of $[r] \backslash\{g\}$, then

$$
v_{I}^{b}=\ell(b)+1+\alpha \cdot a_{I \cup\{g\}}(b) .
$$

But, as explained above, $a_{I}(b)=\sum_{i \in I} a_{i}(b)$. Thus $v_{I}^{b}$ is as in Lemma 3.5 with the following values of the parameters: $K=[r] \backslash\{g\}, C=\ell(b)+1+\alpha a_{g}(b)$, and $c_{i}=a_{i}(b)$ for $i \neq g$. Therefore the lemma asserts that $T_{[r] \backslash\{g\}}\left(\boldsymbol{v}^{b}\right)=O\left(\alpha^{r-1}\right)$. Going back to Eq. [12], we have:

$$
1+T_{[r]}(\boldsymbol{u})=\prod_{g \in[r]} \prod_{b \in \lambda^{g}}\left(1+T_{[r] \backslash\{g\}}\left(\boldsymbol{v}^{b}\right)\right)=1+O\left(\alpha^{r-1}\right),
$$

which completes the proof.

Let us now look at the second hook-polynomial $h_{\alpha}^{\prime}$. If we try to adapt the argument above, we want, for each box $b$, to apply Lemma 3.5 with $K=[r] \backslash\{g\}, C=\ell(b)+\alpha\left(1+a_{g}(b)\right)$, and $c_{i}=a_{i}(b)$ for $i \neq g$. But boxes $b$ with leg-length 0 will create a difficulty as, for such boxes, $C^{-1}$ is not $O(1)$ and the hypothesis of Lemma 3.5 is not fulfilled. To overcome this problem we define

$$
h_{\alpha}^{\prime \prime}(\lambda)=\prod_{\substack{\square \in \lambda \\ \ell(\square) \neq 0}}(\alpha a(\square)+\ell(\square)+\alpha), \text { so that } h_{\alpha}^{\prime}(\lambda)=\alpha^{\lambda_{1}}\left(\prod_{i} m_{i}\left(\lambda^{t}\right) !\right) h_{\alpha}^{\prime \prime}(\lambda) .
$$

Then, the exact same proof as for $h_{\alpha}$ yields the following result:

Proposition 3.7 Fix some partitions $\lambda^{1}, \ldots, \lambda^{r}$ and, for any subset I of $[r]$, set $v_{I}:=h_{\alpha}^{\prime \prime}\left(\lambda^{I}\right)$. The family $\left(v_{I}\right)$ has the strong factorization, and hence, the small cumulant properties.

\section{Strong factorization property of Jack polynomials}

Let us fix partitions $\lambda^{1}, \ldots, \lambda^{r}$, and for any subset $I \subseteq[r]$ we define $u_{I}:=J_{\lambda^{I}}^{(\alpha)}$; we will consider this particular family $\boldsymbol{u}=\left(u_{I}\right)_{I \subseteq[r]}$ throughout this section. With this choice, the cumulant $\kappa_{[r]}(\boldsymbol{u})$ is equal to the cumulant $\kappa^{J}\left(\lambda^{1}, \cdots, \lambda^{r}\right)$ considered in the introduction. The purpose of this section is to prove Theorem 1.4 namely that $\kappa_{[r]}(\boldsymbol{u})$ is $O\left(\alpha^{r-1}\right)$.

\subsection{Preliminary results}

Proposition 4.1 For any partitions $\lambda^{1}, \ldots, \lambda^{r}$ there exists coefficients $c_{\mu}^{\lambda^{1}, \ldots, \lambda^{r}} \in \mathbb{Q}[\alpha]$ such that

$$
\kappa_{[r]}(\boldsymbol{u})=\sum_{\mu<\lambda^{[r]}} c_{\mu}^{\lambda^{1}, \ldots, \lambda^{r}} m_{\mu}+O\left(\alpha^{r-1}\right) .
$$

Proof: First observe that for any partitions $\nu^{1}$ and $\nu^{2}$, we have

$$
m_{\nu^{1}} m_{\nu^{2}}=m_{\nu^{1} \oplus \nu^{2}}+\sum_{\mu<\nu^{1} \oplus \nu^{2}} b_{\mu}^{\nu^{1}, \nu^{2}} m_{\mu},
$$

for some integers $b_{\mu}^{\nu^{1}, \nu^{2}}$. Thanks to property (C2) from Proposition 2.1 and the above observation on products of monomials, for any set-partition $\pi=\left\{\pi_{1}, \cdots, \pi_{s}\right\} \in \mathcal{P}([r])$, there exist coefficients $d_{\mu}^{\lambda^{\pi_{1}}, \cdots, \lambda^{\pi_{s}}} \in \mathbb{Q}[\alpha]$ such that:

$$
J_{\lambda^{\pi_{1}}}^{(\alpha)} \cdots J_{\lambda^{\pi_{s}}}^{(\alpha)}=h_{\alpha}\left(\lambda^{\pi_{1}}\right) \cdots h_{\alpha}\left(\lambda^{\pi_{s}}\right) m_{\lambda^{[r]}}+\sum_{\mu<\lambda^{[r]}} d_{\mu}^{\lambda^{\pi_{1}}, \cdots, \lambda^{\pi_{s}}} m_{\mu}
$$


As a consequence, there exist coefficients $c_{\mu}^{\lambda^{1}, \ldots, \lambda^{r}} \in \mathbb{Q}[\alpha]$ such that

$$
\kappa_{[r]}(\boldsymbol{u})=\kappa_{[r]}(\boldsymbol{v}) m_{\lambda^{[r]}}+\sum_{\mu<\lambda^{[r]}} c_{\mu}^{\lambda^{1}, \ldots, \lambda^{r}} m_{\mu},
$$

where $v_{I}=h_{\alpha}\left(\lambda^{I}\right)$. Proposition 3.6 completes the proof.

Let us now define two functions that will be of great importance in the proof of Theorem 1.4

$$
\begin{aligned}
& A_{1}\left(\lambda^{1}, \ldots, \lambda^{r}\right):=\sum_{\pi \in \mathcal{P}([r])}\left[(-1)^{\#(\pi)-1}(\#(\pi)-1) !\left(\sum_{B \in \pi} b\left(\lambda^{B}\right)\right) \prod_{B \in \pi} J_{\lambda^{B}}^{(\alpha)}\right], \\
& A_{2}\left(\lambda^{1}, \ldots, \lambda^{r}\right):=\sum_{\pi \in \mathcal{P}([r]) ; \#(\pi) \geq 2}(-1)^{\#(\pi)-1}(\#(\pi)-1) ! D_{1,2}\left(J_{\lambda^{B}}^{(\alpha)}: B \in \pi\right),
\end{aligned}
$$

where $D_{1,2}$ is defined by

$$
D_{1,2}\left(f_{1}, \ldots, f_{k}\right):=\sum_{1 \leq m \leq N} \sum_{1 \leq i<j \leq k} f_{1} \cdots\left(x_{m} \frac{\partial}{\partial x_{m}} f_{i}\right) \cdots\left(x_{m} \frac{\partial}{\partial x_{m}} f_{j}\right) \cdots f_{k} .
$$

Given two partitions $\lambda^{1}$ and $\lambda^{2}$ we also define $\operatorname{InEx}\left(\lambda^{1}, \lambda^{2}\right):=b\left(\lambda^{1} \oplus \lambda^{2}\right)-b\left(\lambda^{1}\right)-b\left(\lambda^{2}\right)$. Here is a key lemma (for a proof, we refer to [2, Lemmas 4.3 and 4.4]) that expresses $A_{1}$ and $A_{2}$ in terms of cumulants.

Lemma 4.2 For any positive integer $r \geq 2$ and any partitions $\lambda^{1}, \ldots, \lambda^{r}$ we have the following equalities:

$$
\begin{aligned}
& A_{1}\left(\lambda^{1}, \ldots, \lambda^{r}\right)=b\left(\lambda^{[r]}\right) \kappa_{[r]}(\boldsymbol{u})+\frac{1}{2} \sum_{\emptyset \subsetneq I \subsetneq[r]} \operatorname{InEx}\left(\lambda^{I}, \lambda^{I^{c}}\right) \kappa_{I}(\boldsymbol{u}) \kappa_{I^{c}}(\boldsymbol{u}), \\
& A_{2}\left(\lambda^{1}, \ldots, \lambda^{r}\right)=-\frac{1}{2} \sum_{1 \leq m \leq N} \sum_{\emptyset \subsetneq I \subsetneq[r]}\left(x_{m} \frac{\partial}{\partial x_{m}} \kappa_{I}(\boldsymbol{u})\right)\left(x_{m} \frac{\partial}{\partial x_{m}} \kappa_{I^{c}}(\boldsymbol{u})\right),
\end{aligned}
$$

where $I^{c}$ denotes the complement of $I$ in $[r]$, i.e. $I^{c}:=[r] \backslash I$.

\subsection{Proof of Theorem 1.4}

Proof of Theorem 1.4; The proof will by given by induction on $r$. Observe that the case $r=2$ follows from the specialization of Jack polynomials at $\alpha=0$.

Let us assume that the statement holds true for all $m<r$. Notice first that by Leibniz rule, for any $f_{1}, \ldots, f_{k} \in$ Sym, one has the following expansions:

$$
D_{\alpha}\left(f_{1} \cdots f_{k}\right)=\sum_{1 \leq i \leq k} f_{1} \cdots\left(D_{\alpha} f_{i}\right) \cdots f_{k}+D_{1,2}\left(f_{1}, \ldots, f_{k}\right),
$$

where $D_{1,2}$ is given by Eq. 16 . Fix some partitions $\lambda^{1}, \ldots, \lambda^{r}$ and a set-partition $\pi=\left\{\pi_{1}, \ldots, \pi_{s}\right\}$ of $[r]$. Then applying above Leibniz rule, and Proposition 2.1 one has

$$
\begin{aligned}
D_{\alpha}\left(J_{\lambda^{\pi_{1}}}^{(\alpha)} \cdots J_{\lambda^{\pi_{s}}}^{(\alpha)}\right) & =\left((N-1)\left|\lambda^{[r]}\right|-b\left(\left(\lambda^{[r]}\right)^{t}\right)\right) J_{\lambda^{\pi_{1}}}^{(\alpha)} \cdots J_{\lambda^{\pi_{s}}}^{(\alpha)} \\
& +\alpha\left(\left(\sum_{1 \leq i \leq s} b\left(\lambda^{\pi_{i}}\right)\right) J_{\lambda^{\pi_{1}}}^{(\alpha)} \cdots J_{\lambda^{\pi_{s}}}^{(\alpha)}+D_{1,2}\left(J_{\lambda^{\pi_{1}}}^{(\alpha)}, \ldots, J_{\lambda^{\pi_{s}}}^{(\alpha)}\right)\right) .
\end{aligned}
$$


Using now the definition of cumulants, (8), it gives us the following identity:

$$
D_{\alpha} \kappa_{[r]}(\boldsymbol{u})=\left((N-1)\left|\lambda^{[r]}\right|-b\left(\left(\lambda^{[r]}\right)^{t}\right)\right) \kappa_{[r]}(\boldsymbol{u})+\alpha\left(A_{1}\left(\lambda^{1}, \ldots, \lambda^{r}\right)+A_{2}\left(\lambda^{1}, \ldots, \lambda^{r}\right)\right)
$$

where $A_{1}$ and $A_{2}$ are given by (14) and $(15)$, respectively. Consider the coefficient of $\alpha^{j}$ in the expression above. We have

$$
\begin{aligned}
{\left[\alpha^{j}\right] D_{\alpha} \kappa_{[r]}(\boldsymbol{u})=\left((N-1)\left|\lambda^{[r]}\right|-b\left(\left(\lambda^{[r]}\right)^{t}\right)\right)\left[\alpha^{j}\right] \kappa_{[r]}(\boldsymbol{u}) } & \\
& +\left[\alpha^{j-1}\right]\left(A_{1}\left(\lambda^{1}, \ldots, \lambda^{r}\right)+A_{2}\left(\lambda^{1}, \ldots, \lambda^{r}\right)\right)
\end{aligned}
$$

On the other hand, since $D_{\alpha}=D_{1}+\alpha D_{2}$, one has

$$
\left[\alpha^{j}\right] D_{\alpha} \kappa_{[r]}(\boldsymbol{u})=D_{1}\left(\left[\alpha^{j}\right] \kappa_{[r]}(\boldsymbol{u})\right)+D_{2}\left(\left[\alpha^{j-1}\right] \kappa_{[r]}(\boldsymbol{u})\right)
$$

Comparing both expression, we have the following identity, which will be a key tool in the proof:

$$
\begin{aligned}
& D_{1}\left(\left[\alpha^{j}\right] \kappa_{[r]}(\boldsymbol{u})\right)+D_{2}\left(\left[\alpha^{j-1}\right] \kappa_{[r]}(\boldsymbol{u})\right) \\
& \quad=\left((N-1)\left|\lambda^{[r]}\right|-b\left(\left(\lambda^{[r]}\right)^{t}\right)\right)\left[\alpha^{j}\right] \kappa_{[r]}(\boldsymbol{u})+\left[\alpha^{j-1}\right]\left(A_{1}\left(\lambda^{1}, \ldots, \lambda^{r}\right)+A_{2}\left(\lambda^{1}, \ldots, \lambda^{r}\right)\right)
\end{aligned}
$$

We recall that our goal is to prove that $\left[\alpha^{j}\right] \kappa_{[r]}(\boldsymbol{u})=0$ for any $0 \leq j \leq r-2$. We proceed by induction on $j$. Consider the case $j=0$. Since $\kappa_{[r]}(\boldsymbol{u}), A_{1}$ and $A_{2}$ are polynomials in $\alpha$, Eq. 19. simplifies then to

$$
D_{1} f=\left((N-1)\left|\lambda^{[r]}\right|-b\left(\left(\lambda^{[r]}\right)^{t}\right)\right) f,
$$

where $f=\left[a^{0}\right] \kappa_{[r]}(\boldsymbol{u})$. Thanks to Proposition 4.1 we know that $f$ satisfies the assumptions of Corollary 2.3 and hence it is equal to zero.

Now, we fix $j \leq r-2$ and assume that $\left[\alpha^{i}\right] \kappa_{[r]}(\boldsymbol{u})=0$ holds true for all $0 \leq i<j$; we are going to show that it holds true for $i=j$ as well.

First, we claim that $\left[\alpha^{j-1}\right] A_{1}\left(\lambda^{1}, \ldots, \lambda^{r}\right)=0$. Indeed, from the induction hypothesis, for each subset $I$ with $\emptyset \subsetneq I \subsetneq[r]$ one has $\kappa_{I}(\boldsymbol{u})=O\left(\alpha^{|I|-1}\right)$ and $\kappa_{I^{c}}(\boldsymbol{u})=O\left(\alpha^{\left|I^{c}\right|-1}\right)=O\left(\alpha^{r-|I|-1}\right)$. We then use Lemma 4.2 and write (recall that $j-1<r-2$ ):

$$
\left[\alpha^{j-1}\right] A_{1}\left(\lambda^{1}, \ldots, \lambda^{r}\right)=b\left(\lambda^{[r]}\right)\left[\alpha^{j-1}\right] \kappa_{[r]}(\boldsymbol{u})+\frac{1}{2} \sum_{\emptyset \subsetneq I \subsetneq[r]}\left[\alpha^{j-1}\right] \operatorname{InEx}\left(\lambda^{I}, \lambda^{I^{c}}\right) \kappa_{I}(\boldsymbol{u}) \kappa_{I^{c}}(\boldsymbol{u})=0 .
$$

Similarly, one can prove that $\left[\alpha^{j-1}\right] A_{2}\left(\lambda^{1}, \ldots, \lambda^{r}\right)=0$. Indeed, using a similar argument as before, we have

$$
\frac{1}{2} \sum_{1 \leq m \leq N} \sum_{\emptyset \subsetneq I \subsetneq[r]}\left(x_{m} \frac{\partial}{\partial x_{m}} \kappa_{I}(\boldsymbol{u})\right)\left(x_{m} \frac{\partial}{\partial x_{m}} \kappa_{I^{c}}(\boldsymbol{u})\right)=O\left(\alpha^{r-2}\right) .
$$

But, from Eq. (18), the left-hand side is $A_{2}\left(\lambda^{1}, \ldots, \lambda^{r}\right)$, and we have $j-1<r-2$, which implies that $\left[\alpha^{j-1}\right] A_{2}\left(\lambda^{1}, \ldots, \lambda^{r}\right)=0$, as wanted.

Moreover, by induction hypothesis, $\left[\alpha^{j-1}\right] \kappa_{[r]}(\boldsymbol{u})=0$. These computations show that Eq. (19] simplifies to

$$
D_{1} f=\left((N-1)\left|\lambda^{[r]}\right|-b\left(\left(\lambda^{[r]}\right)^{t}\right)\right) f
$$

where $f=\left[a^{j}\right] \kappa_{[r]}(\boldsymbol{u})$. Again, thanks to Proposition 4.1 we know that $f$ satisfies assumptions from Corollary 2.3 and thus is equal to zero, which finishes the proof. 


\section{Polynomiality in $b$-conjecture}

\subsection{Cumulants of functions on Young diagrams}

Consider a function $F$ on Young diagrams and some diagrams $\lambda^{1}, \cdots, \lambda^{r}$. Then we consider the family defined by $u_{I}=F\left(\bigoplus_{i \in I} \lambda^{i}\right)$ (recall that we use $\oplus$ for entry-wise sum of partitions).

Definition 5.1 We say that a function $G$ on Young diagrams has the small cumulant property if, for any $r \geq 1$ and for any partitions $\lambda^{1}, \cdots, \lambda^{r}$, the above-defined family has the small cumulant property.

With this terminology, the results of the previous sections can be reformulate as:

Theorem 1.4 For a fixed alphabet $\boldsymbol{x}$, the function $\lambda \mapsto J_{\lambda}^{(\alpha)}(\boldsymbol{x})$ has the small cumulant property.

Propositions 3.6 and 3.7 The functions $h_{\alpha}$ and $h_{\alpha}^{\prime \prime}$ have the small cumulant property.

Corollary 3.4 If $G_{1}$ and $G_{2}$ have the small cumulant properties and take non-zero values, then so have $G_{1} \cdot G_{2}$ and $G_{1} / G_{2}$ (if $G_{2}$ is not identically equal to 0 ).

As a consequence, the function $\lambda \mapsto \frac{1}{h_{\alpha}(\lambda) h_{\alpha}^{\prime \prime}(\lambda)} J_{\lambda}^{(\alpha)}(\boldsymbol{x}) J_{\lambda}^{(\alpha)}(\boldsymbol{y}) J_{\lambda}^{(\alpha)}(\boldsymbol{z})$ has the small cumulant property. We will use that later in this Section.

\subsection{Cumulants and logarithm}

Lemma 5.2 Let $F$ be a function on Young diagrams and define $\boldsymbol{u}$ as above. Denote $\kappa^{F}\left(\lambda^{1}, \cdots, \lambda^{r}\right)$ the cumulant $\kappa_{[r]}(\boldsymbol{u})$. Then we have the following equality of formal power series in $\boldsymbol{t}=\left(t_{1}, t_{2}, \ldots\right)$ :

$$
\log \sum_{\lambda} \frac{F(\lambda)}{\alpha^{\lambda_{1}} \prod_{i} m_{i}\left(\lambda^{t}\right) !} \boldsymbol{t}^{\lambda^{t}}=\sum_{r \geq 1} \frac{1}{r ! \alpha^{r}} \sum_{\left(j_{1}, \cdots, j_{r}\right)} \kappa^{F}\left(1^{j_{1}}, \cdots, 1^{j_{r}}\right) t_{j_{1}} \cdots t_{j_{r}} .
$$

Proof: Omitted. The proof consist in comparing the coefficient of $F\left(\lambda^{1}\right) \cdots F\left(\lambda^{s}\right) \boldsymbol{t}^{\left(\lambda^{1}\right)^{t}} \cdots \boldsymbol{t}^{\left(\lambda^{s}\right)^{t}}$ in both sides of the identity we want to establish. See the long version [2] for details.

Remark 5.3 The statement of this lemma is reminiscent of the fact that cumulants can be either defined as a sum over set-partitions or as coefficients in the generating series of the logarithm of the moment generating series; see, e.g. Eqs (3) and (II.c) in [13]. The proof is also an easy adaptation of the one of this well-understood statement.

We have now all the tools needed to prove the polynomiality in $b$-conjecture.

Proof of Theorem 1.2; Recall that it is enough to prove that $h_{\mu, \nu}^{\tau}(\beta)$ has no pole in $\alpha=0$, i.e. that $h_{\mu, \nu}^{\tau}(\beta)=O(1)$. From Eq. (1), this amounts to establish that

$$
\log \left(\sum_{\tau \in \mathcal{P}} \frac{J_{\tau}^{(\alpha)}(\boldsymbol{x}) J_{\tau}^{(\alpha)}(\boldsymbol{y}) J_{\tau}^{(\alpha)}(\boldsymbol{z}) t^{|\tau|}}{h_{\alpha}(\lambda) h_{\alpha}^{\prime}(\lambda)}\right)=O\left(\alpha^{-1}\right) .
$$

But, using Eq. 13, we see that this quantity is the left-hand side of Lemma 5.2 for

$$
F(\lambda)=\frac{1}{h_{\alpha}(\lambda) h_{\alpha}^{\prime \prime}(\lambda)} J_{\lambda}^{\alpha}(\boldsymbol{x}) J_{\lambda}^{\alpha}(\boldsymbol{y}) J_{\lambda}^{\alpha}(\boldsymbol{z})
$$

and $t_{1}=t_{2}=\cdots=t$. It was observed at the end of Section 5.1 that this function $F$ has the small cumulant property. Therefore, for any $j_{1}, \cdots, j_{r}$, the cumulant $\kappa^{F}\left(1^{j_{1}}, \cdots, 1^{j_{r}}\right)$ is $O\left(\alpha^{r-1}\right)$ and, thus, the right-hand side of Lemma 5.2 is $O\left(\alpha^{-1}\right)$. This finishes the proof of the polynomiality. 


\section{References}

[1] D. R. L. Brown and D. M. Jackson. A rooted map invariant, non-orientability and Jack symmetric functions. J. Combin. Theory Ser. B, 97(3):430-452, 2007.

[2] M. Dołega and V. Féray. Cumulants of Jack symmetric functions and $b$-conjecture. Preprint arXiv:1601.01501, 2016.

[3] M. Dołęga and V. Féray. Gaussian fluctuations of Young diagrams and structure constants of Jack characters. Duke Math. J., Advance Publication, 2016. doi: 10.1215/00127094-3449566.

[4] M. Dołęga, V. Féray, and P. Śniady. Jack polynomials and orientability generating series of maps. Sém. Lothar. Combin., 70:Art. B70j, 50 pp. (electronic), 2014.

[5] V. Féray. Asymptotic behavior of some statistics in Ewens random permutations. Electron. J. Probab, 18(76):1-32, 2013.

[6] I. P. Goulden and D. M. Jackson. Connection coefficients, matchings, maps and combinatorial conjectures for Jack symmetric functions. Trans. Amer. Math. Soc., 348(3):873-892, 1996.

[7] A. L. Kanunnikov and E. A. Vassilieva. A recurrence formula for Jack connection coefficients. preprint arXiv:1409.4356, 2014.

[8] F. Knop and S. Sahi. A recursion and a combinatorial formula for Jack polynomials. Inv. Math., 128(1):9-22, 1997.

[9] M. A. La Croix. The combinatorics of the Jack parameter and the genus series for topological maps. $\mathrm{PhD}$ thesis, University of Waterloo, 2009.

[10] S. K. Lando and A. K. Zvonkin. Graphs on surfaces and their applications, volume 141 of Encyclopaedia of Mathematical Sciences. Springer-Verlag, Berlin, 2004. With an appendix by Don B. Zagier.

[11] M. Lassalle. A positivity conjecture for Jack polynomials. Math. Res. Lett., 15(4):661-681, 2008.

[12] M. Lassalle. Jack polynomials and free cumulants. Adv. Math., 222(6):2227-2269, 2009.

[13] V. Leonov and A. Shiryaev. On a method of calculation of semi-invariants. Th. Prob. Appl., 4(3):319$329,1959$.

[14] I. G. Macdonald. Symmetric functions and Hall polynomials. Oxford Mathematical Monographs. The Clarendon Press Oxford University Press, New York, second edition, 1995. With contributions by A. Zelevinsky, Oxford Science Publications.

[15] R. P. Stanley. Some combinatorial properties of Jack symmetric functions. Adv. Math., 77(1):76$115,1989$. 